\title{
Estudio de caso del aprendizaje basado en proyectos desde los actores de nivel primaria
} Case study of project-based learning from primary level actors

\section{Estudo de caso de aprendizagem baseada em projetos de atores de nível primário}

María Obdulia González-Fernández

Universidad de Guadalajara, México ogonzalez@cualtos.udg.mx https://orcid.org/0000-0001-5890-7666

Laura Becerra Vázquez

Universidad Pedagógica Nacional UPN 142, México

laurabecerravazquez498@gmail.com http://orcid.org/0000-0003-0832-749X

\section{Resumen}

Este trabajo presenta los resultados de una investigación cualitativa con la finalidad de caracterizar los desafíos de la metodología de aprendizaje basado en problemas (ABP) en una institución privada de educación básica en México. El estudio se desarrolló a partir de 15 entrevistas realizadas a directivos, docentes y alumnos. Se utilizó el análisis de contenido para la sistematización de los datos. Los códigos principales fueron percepción del aprendizaje, limitaciones, sugerencias, ventajas y ambiente de aprendizaje. Los resultados demuestran que la metodología de aprendizaje basado en proyectos promueve el pensamiento crítico, la creatividad y la autonomía. Como uno de los desafíos se encuentran los paradigmas tradicionales, el conformismo y el desconocimiento de la estrategia por parte de los docentes. En específico, los resultados de las entrevistas a estudiantes demuestran que no cuentan con las habilidades para el manejo de la información al momento de generar los proyectos; además, necesitan reforzar las habilidades para la coordinación del trabajo en el interior de un equipo. Se destaca la importancia del generar ambientes de colaboración, para lo cual la temática o pregunta del proyecto debe ser motivadora para el estudiante y, por último, promover en todo momento una actitud activa frente el aprendizaje. Estos resultados permiten conocer una 


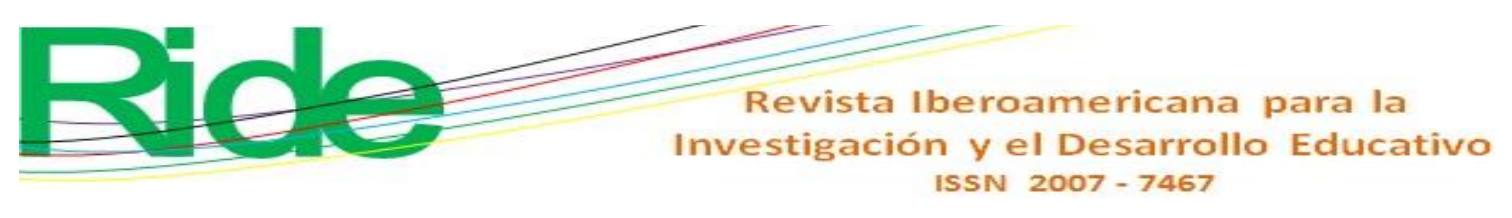

parte de la integración en el nivel básico del ABP, por lo que ayuda a impulsar su integración en las diferentes comunidades educativas, no solo de forma enunciativa, sino práctica.

Por último, como limitantes del estudio se identifica el carácter metodológico, por lo que para futuras indagaciones de este tipo se puede aumentar la muestra, lo que permitirá conocer la realidad de aplicar dicha metodología en otras instituciones.

Palabras clave: aprendizaje activo, aprendizaje basado en proyectos, innovación educativa, método de enseñanza, modelo educativo, nivel primario.

\begin{abstract}
This paper presents the results of a qualitative research in order to characterize the challenges of the Problem-Based Learning (PBL) methodology in a private institution of basic education in Mexico. The study is qualitative in nature, based on 15 interviews with managers, teachers and students. Content analysis was used to systematize the data. The main codes were: Perception of learning, limitations, suggestions, advantages and learning environment.

The results show that the project-based learning methodology mainly promotes critical thinking, creativity and autonomy. As one of the challenges is the traditional paradigms, conformity and ignorance on the part of teachers. Specifically, the results of the interviews with student's state that they do not have the skills to manage information at the time of generating the projects, in addition to the fact that it is necessary to reinforce the skills for the coordination of work within a team. The importance of generating collaborative environments is highlighted, that the theme or question of the project is motivating for the student and finally, promoting an active attitude towards learning at all times. These results allow us to know one face of integration at the basic level of PBL, thus helping to promote its integration in the different educational communities, not only in an enunciative but also in a practical way. Finally, as limitations of the study, the methodological nature is identified, so that for future studies of this type the sample can be increased that allows knowing the situation of the application of said methodology in other institutions.
\end{abstract}

Keywords: activity learning, project-based learning, educational innovations, teaching methods, educational models, elementary education. 


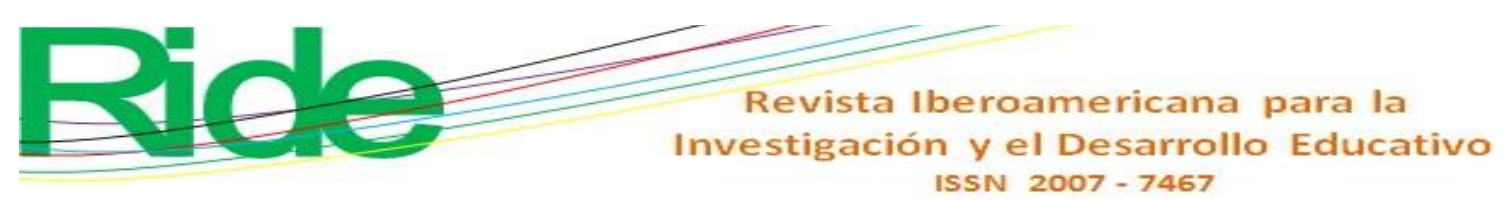

\section{Resumo}

Este artigo apresenta os resultados de uma pesquisa qualitativa com o objetivo de caracterizar os desafios da metodologia de aprendizagem baseada em problemas (PBL) em uma instituição privada de educação básica no México. O estudo foi desenvolvido a partir de 15 entrevistas com gestores, professores e alunos. A análise de conteúdo foi utilizada para sistematizar os dados. Os principais códigos foram percepção de aprendizagem, limitações, sugestões, vantagens e ambiente de aprendizagem. Os resultados mostram que a metodologia de aprendizagem baseada em projetos promove o pensamento crítico, a criatividade e a autonomia. Como um dos desafios estão os paradigmas tradicionais, o conformismo e o desconhecimento da estratégia por parte dos professores. Especificamente, os resultados das entrevistas com os alunos mostram que eles não possuem habilidades para lidar com as informações na hora de gerar os projetos; além disso, eles precisam reforçar as habilidades para coordenar o trabalho em uma equipe. Destaca-se a importância de gerar ambientes colaborativos, para os quais o tema ou questão do projeto deve ser motivador para o aluno e, por fim, promover uma atitude ativa em relação à aprendizagem em todos os momentos. Esses resultados nos permitem conhecer um pouco da integração no nível básico do PBL, ajudando assim a promover sua integração nas diferentes comunidades educacionais, não só de forma enunciativa, mas também na prática.

Por fim, como limitações do estudo, identifica-se a natureza metodológica, de modo que para futuras investigações deste tipo a amostra possa ser ampliada, o que permitirá conhecer a realidade de aplicação desta metodologia em outras instituições.

Palavras-chave: aprendizagem ativa, aprendizagem baseada em projetos, inovação educacional, método de ensino, modelo educacional, nível primário.

Fecha Recepción: Septiembre 2020

Fecha Aceptación: Febrero 2021

\section{Introducción}

La educación del siglo XXI debe adaptarse a los escenarios actuales y a las nuevas formas de aprender de los estudiantes, quienes exigen métodos de enseñanza basados en modelos pedagógicos activos. De acuerdo con García Pérez (2000), el renovar la realidad educativa parte de una profunda reflexión desde su modelo didáctico deseable, así como de la organización y dinámicas reales de los centros educativos. Así se destacan los modelos didácticos que centran su atención en mecanismos para favorecer la construcción de conocimientos a partir de proyectos y problemas. 


\section{El aprendizaje basado en proyectos}

Una forma de llevar a cabo los métodos activos es a través del aprendizaje basado en proyectos $(\mathrm{ABP})$ o aprendizaje orientado a proyectos (AOP) (project-based learning o PBL), que beneficia el trabajo activo del estudiante y el trabajo colaborativo a partir de la negociación de los participantes (García-Varcálcel, Muñoz-Repiso y Gómez-Pablos, 2017). Araújo y Sastre (2018) plantean que esta metodología se está perfilando como uno de los enfoques innovadores en la formación académica actual. Perrenoud (1999) y Trujillo (2015) la reconocen como una de las metodologías activas, ya que a partir de sus fases "el proyecto" es la espina dorsal de una pedagogía para la construcción de saberes en una clase. Sus objetivos se enfocan en lograr movilizar saberes o procedimientos en pro de la construcción de competencias, además de incrementar el sentido de los saberes y de los aprendizajes escolares o, en su defecto, describir nuevos saberes, perspectivas y propiciar la motivación en el marco del proyecto a partir de la cooperación entre compañeros.

El aprendizaje basado en proyectos fomenta una forma de trabajo que privilegia la investigación, a partir de diversas interrogantes planteadas a los estudiantes, los cuales deben procurar resolver mediante la búsqueda de información en diversas fuentes. De este modo se favorece la indagación de una realidad movilizando conceptos al confrontar información (Martín y Rodríguez, 2015).

Estas estrategias en la actualidad han tomado mayor fuerza a raíz de los modelos por competencias impulsados por los sistemas educativos mundiales que buscan que el estudiante logre desarrollar las habilidades y actitudes para realizar una tarea. En este contexto, el proyecto se asocia a cualquier acción productiva que exige la articulación de actividades para generar productos, resolver problemas o satisfacer necesidades e inquietudes, considerando los recursos y el tiempo asignado. De acuerdo con Díaz Barriga (2006), en la conducción de un proyecto, los alumnos participan de forma productiva y colaborativa a favor de la construcción del conocimiento de una manera innovadora. En la metodología por proyectos el estudiante realiza una resignificación del proceso de enseñanza-aprendizaje, ya que permite crear dinámicas de cooperación que enfrentan al estudiante a situaciones que lo llevan a plantear propuestas ante determinada situación.

La característica del ABP es la construcción del conocimiento a partir de la globalidad del proyecto, la necesidad de investigación en diferentes fuentes, el trabajo colaborativo, la unión entre la realidad y los contenidos trabajados en la escuela, así como las relaciones entre alumnos-alumnos, alumno-profesor, familia y entorno, lo que aumenta la motivación (Barba-Marín, Sonlleva-Velasco y García-Marín, 2018). 


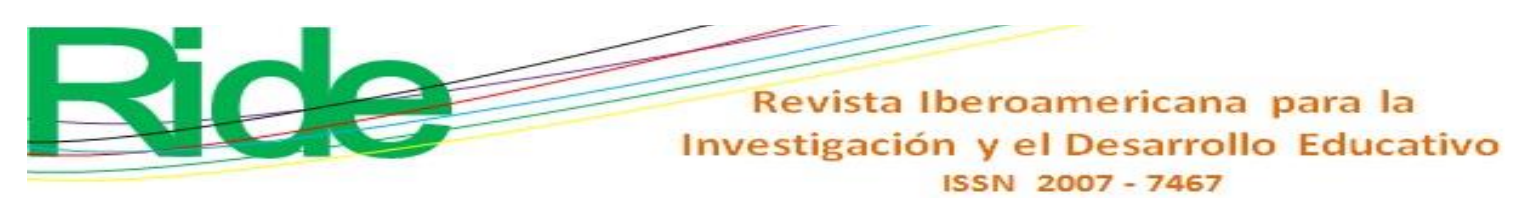

Este último paso se puede realizar a partir de mecanismos de coevaluación, autoevaluación y heteroevaluación para que alumnos y docentes cierren el proyecto con procesos metacognitivos de lo vivido. Por ende, es importante contar con instrumentos como rúbricas y listas de cotejo.

El papel activo tanto del docente y como del estudiante está presente en cada una de las etapas del desarrollo del proyecto, por lo que se recomienda que sea enmarcado por los enfoques de referencia de las asignaturas implicadas y que se cuente con el acompañamiento del docente durante todo el proceso del proyecto.

Son muchas las ventajas que este modelo ofrece al proceso de aprendizaje, ya que promueve que los estudiantes piensen y actúen con base en el diseño de un proyecto, elaborando un plan con estrategias definidas para dar una solución a una interrogante al fomentar la indagación de los estudiantes. Al trabajar con proyectos, los alumnos exploran y descubren sus intereses, ponen en común sus concepciones y comparten información (García-Varcálcel et al., 2017). Por tanto, permite el aprender en la diversidad al trabajar todos juntos, estimula el crecimiento emocional, intelectual y personal mediante experiencias directas con personas y estudiantes ubicados en diferentes contextos.

Los estudiantes, en definitiva, aprenden diferentes técnicas para la solución de problemas al estar en contacto con personas de diversas culturas y con puntos de vista diferentes; en otras palabras, aprenden a aprender el uno del otro y también aprenden la forma de ayudar a que sus compañeros aprendan. Asimismo, aprenden a evaluar el trabajo de sus pares y a dar retroalimentación constructiva tanto para ellos mismos como para sus compañeros. Las metodologías activas como el aprendizaje basado en proyectos se promueve el trabajo en equipo, donde el estudiante es el protagonista, por lo general desarrolla un producto en conjunto para el logro de un objetivo común (Rodríguez, Vargas y Luna, 2010). El papel del docente es ser guía, orientar el trabajo y promover las relaciones entre los actores del equipo (Fernández et al., 2018). Por lo tanto, el proceso de elaborar un proyecto alienta a los estudiantes a experimentar, realizar aprendizaje basado en descubrimientos, aprender de sus errores y enfrentar y superar retos difíciles e inesperados.

\section{Experiencia en México del modelo basado en proyectos}

Como ya se mencionó, el caso específico del ABP no es una propuesta nueva, pues la Secretaría de Educación Pública (SEP), en la década de los noventa, ya proponía principios constructivistas en el caso específico del programa de Educación Preescolar Plan 92, que fundamenta las condiciones y organización del trabajo en dicho nivel educativo. Este programa propone la metodología por proyectos bajo el principio 


\section{Metodología}

El objetivo de la presente investigación fue caracterizar las diferentes experiencias del proceso de enseñanza-aprendizaje en el marco innovador de la metodología del aprendizaje basado en proyectos (ABP). Con esto se procuró detectar las bondades de dicha metodología y las áreas de oportunidad para establecer nuevas líneas de investigación que ayuden a los profesores a mejorar su práctica docente. Para ello, se desarrolló un estudio de carácter cualitativo interpretativo, el cual permite indagar situaciones en su ambiente natural para tratar de interpretar los fenómenos (Durán, 2012).

Como técnica se utilizó el estudio de caso, ya que la investigación se circunscribe en un contexto específico de una institución educativa determinada. De acuerdo con Muñiz (2010), en el estudio de caso se considera la selección de una muestra cualitativa para estudiar un fenómeno o evento determinado, por lo que su selección depende de lo que se pretende analizar. En otras palabras, el estudio de caso es una investigación empírica que estudia un fenómeno dentro de su contexto real, por lo que centra su atención en la descripción y examen comprensivo y sistemático del hecho (Durán, 2012).

\section{Población}

La comunidad escolar donde se realizó la investigación es una institución de carácter privado incorporada a la Secretaría de Educación Pública de nivel primaria. Es de reciente creación y cuenta con un horario escolar matutino de 7 horas. Se caracteriza por pertenecer a un nivel socioeconómico medio, medio alto y alto. La comunidad educativa cuenta con 225 estudiantes en el nivel primaria, 11 profesores de grupo y 12 profesores de clases complementarias. Como centro educativo se destaca por ser el ápice de la educación en la región Altos del estado de Jalisco (México), pionero en la implementación de esta metodología para intentar responder a las necesidades del futuro.

\section{Instrumento}

Se elaboró una entrevista semiestructurada a partir de las categorías experiencia del $A B P$, retos de la aplicación del $A B P$ y beneficios del $A B P$. El instrumento fue aplicado a seis docentes, ocho alumnos y un directivo del plantel. Las entrevistas se realizaron durante el año 2019, en el ciclo escolar 2018-2019, mediante grabaciones de audio que contaron con la autorización de la dirección de la institución. 


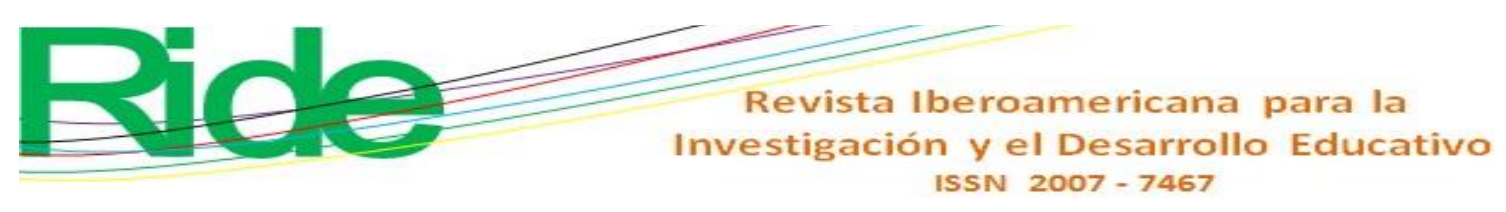

\section{Técnica de análisis de datos}

Para realizar la sistematización de los datos se utilizó la técnica de análisis de contenidos mediante la codificación inductiva de códigos a los cuales se les asignaron etiquetas que indicaban la categoría de una temática o idea. Esta técnica sirve para interpretar escritos y audios que cobran sentido y pueden ser captados dentro de un contexto. En otras palabras, se pueden hacer inferencias válidas y confiables de datos con respecto a un contexto donde los mensajes adquieren significados (Andréu, 2001). El trabajo de análisis siguió los siguientes pasos:

- Determinar el objeto de análisis (la implementación de aprendizaje basado en proyectos).

- Fijar de las reglas de codificación (a partir de las preguntas de la entrevista).

- Elaborar un sistema de categorías de tipo inductivo.

- La codificación, que es la asignación de códigos a cada categoría, clasificando el material para la construcción de un sistema de códigos.

- Generar las inferencias a partir de un inventario en el que se aíslan las unidades de significado, conformando el contenido empírico de las categorías.

Para dicho análisis se utilizó el programa MAXQDA, versión 2018 (software para el análisis de datos cualitativos), en el cual se genera el listado de códigos y segmentos. Asimismo, se asignaron etiquetas para indicar que son ejemplos de alguna idea temática.

\section{Resultados}

Para realizar el análisis de contenido se clasificaron los códigos en cinco categorías: ventajas, limitaciones, ambiente de aprendizaje, aprendizaje y sugerencias. En total, se registraron 80 codificaciones distribuidas de la siguiente forma: 8 correspondientes a la entrevista al directivo, 24 distribuidas en las entrevistas a los alumnos y 48 correspondientes a las entrevistas a los profesores.

\section{Significados del ABP desde la perspectiva docente}

Los resultados de las entrevistas a los profesores demostraron en la categoría aprendizaje que ellos consideran que el aprendizaje por proyecto potencializa la creatividad, la autonomía y el pensamiento crítico. En la categoría ventajas se encontró que la aplicación del conocimiento es uno de los grandes beneficios de dicha forma de trabajo. Al mismo tiempo, se destaca que la metodología genera un ambiente de trabajo colaborativo en grupos. De este modo lo narra un entrevistado: 


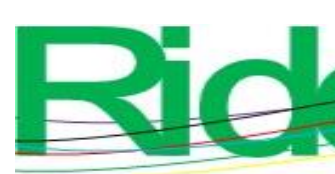

Revista lberoamericana para la

El momento que más propicia el trabajo en equipo es el momento de interacción por medio de los comentarios porque añades tu opinión respecto del contenido del compañero y otros comienzan a opinar por medio de esto (entrevistada 5, maestro, 2019).

Figura 1. Modelo de un caso de la codificación de entrevistas a docentes

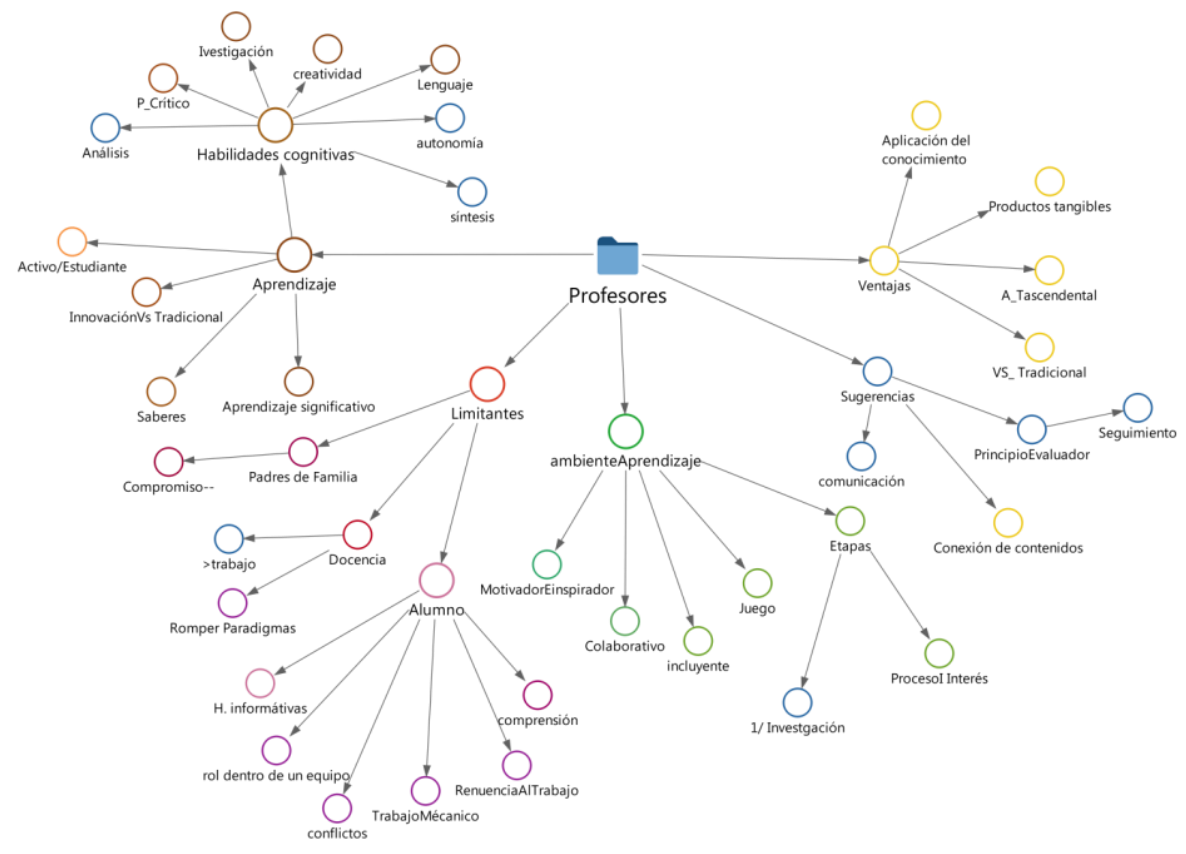

Fuente: Elaboración propia

En la figura 1 se observa la síntesis del análisis de los resultados (el tamaño de los círculos de cada uno de los códigos y subcódigos muestra la frecuencia de las codificaciones). Los docentes consideran que las ventajas del ABP es que desarrolla la creatividad, el pensamiento crítico y las habilidades de investigación. Muestra de ello es el siguiente fragmento de entrevista:

Hace que los alumnos generen un pensamiento crítico y al mismo tiempo un aprendizaje significativo (...). En los niños pequeños desarrolla la creatividad, el ingenio del niño (entrevista 6, maestro, 2019, 1-1:36).

Algunos jóvenes llegan a razonar y logran un objetivo en el proyecto, otros no porque lo hacen de manera mecánica, porque es más lento en unos que en otros, pero sí una buena cantidad logran razonar y aprovechar el proyecto (entrevista 3, maestro, 2019, 42-1:50).

Sin embargo, se reconoce como un reto el trabajo en equipo, como narra el siguiente maestro: 


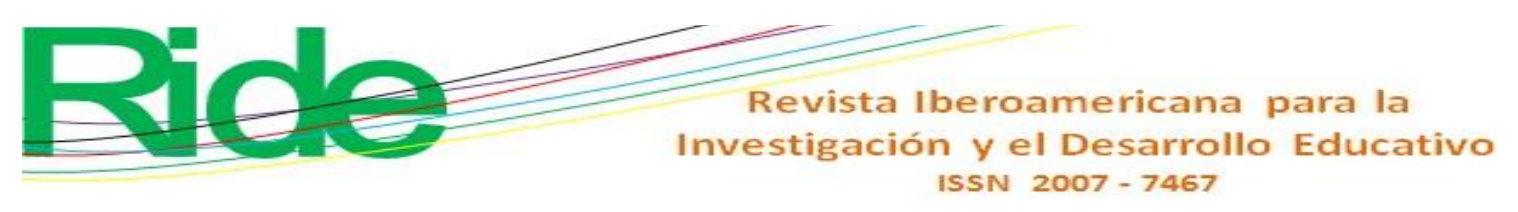

Los retos son si se trabaja en equipo que el alumno logre desempeñar el rol que le toca para el equipo, el ser tolerantes, aceptar las opiniones de los demás y de manera individual que puedan adquirir la información para el proyecto (entrevista 1, maestro, 2019).

Como limitaciones se encontró el compromiso de los padres de familia y su involucración en el trabajo, además de las faltas de habilidades informativas para la búsqueda y procesamiento de información en la etapa inicial, así como la renuencia al trabajo por parte de los estudiantes. Del siguiente modo lo manifestó un entrevistado: "Observo que el alumno se pierde en la información y pierde el objetivo y pueda que se frustre" (entrevistado 1, maestro, 2019).

En resumen, los docentes contemplan el ABP como beneficioso para el aprendizaje de los estudiantes, ya que propicia el desarrollo de habilidades y el romper esquemas tradicionales para transitar a modelos constructivistas. No obstante, también existen limitaciones asociadas con el mayor trabajo de planeación y evaluación. Es necesario, por ende, romper paradigmas educativos tanto en padres de familia como en los mismos alumnos.

\section{La visión del estudiante ante el ABP}

La entrevista realizada a los estudiantes demuestra que perciben mayor aprendizaje con el ABP que con los métodos tradicionales. A su vez, consideran que la metodología permite desarrollar saberes en medio de un ambiente más divertido y grato, lo que llega a ser inspirador. En cuanto a las limitaciones de la metodología, señalan conflictos en el trabajo en equipo. Estos resultados se pueden observar en la figura 2, donde se clasifican las categorías según la jerarquía de códigos (como ya se mencionó, el tamaño del círculo indica la frecuencia de las codificaciones).

Al entrevistar a los alumnos se encontró que el trabajo cooperativo y en equipo les llama la atención:

Las diferencias entre mi anterior escuela y esta es que no tenías el trabajo en equipo y solo éramos individual, y la maestra nos ponía a hacer eso y nos explicaba y ya nos deja hacerlo solo (entrevista 1, alumno, 2019).

Al preguntar sobre las experiencias que han tenido en la realización de proyectos, una alumna comentó que existen conflictos, pero luego se debe llegar a soluciones: "Pues muchos conflictos, pero al final los tenemos que resolver para volvernos a unir" (entrevista 2, alumno, 2019). 
La idea fue porque queríamos trasformar la educación de los Altos de Jalisco; aquí en la zona de Tepatitlán había puro método tradicional... por eso empezamos a generar este tipo de educación constructivista para poder obtener un aprendizaje significativo (entrevista 1, directivo, 2019, 1-17).

Asimismo, en cuanto a los retos menciona lo siguiente:

Cuesta mucho trabajo que los maestros entendieran el cambio del conformismo, yo lo puedo llamar así, ya que el método siempre había estado igual... Los maestros han seguido esa línea sobre todo con los maestros de experiencia, ese choque cultural, con los maestros nuevos no tanto, que se ha venido logrando... Me he enfrentado a problemáticas como que los maestros la lleguen a implementar y comprender el aprendizaje significativo y a que el alumno lo aprenda a desarrollar y que el padre de familia entienda cómo es la metodología (entrevista 1, directivo, 2019, 56-356).

Figura 3. Modelo de jerarquía de códigos de entrevista a directivo del plantel

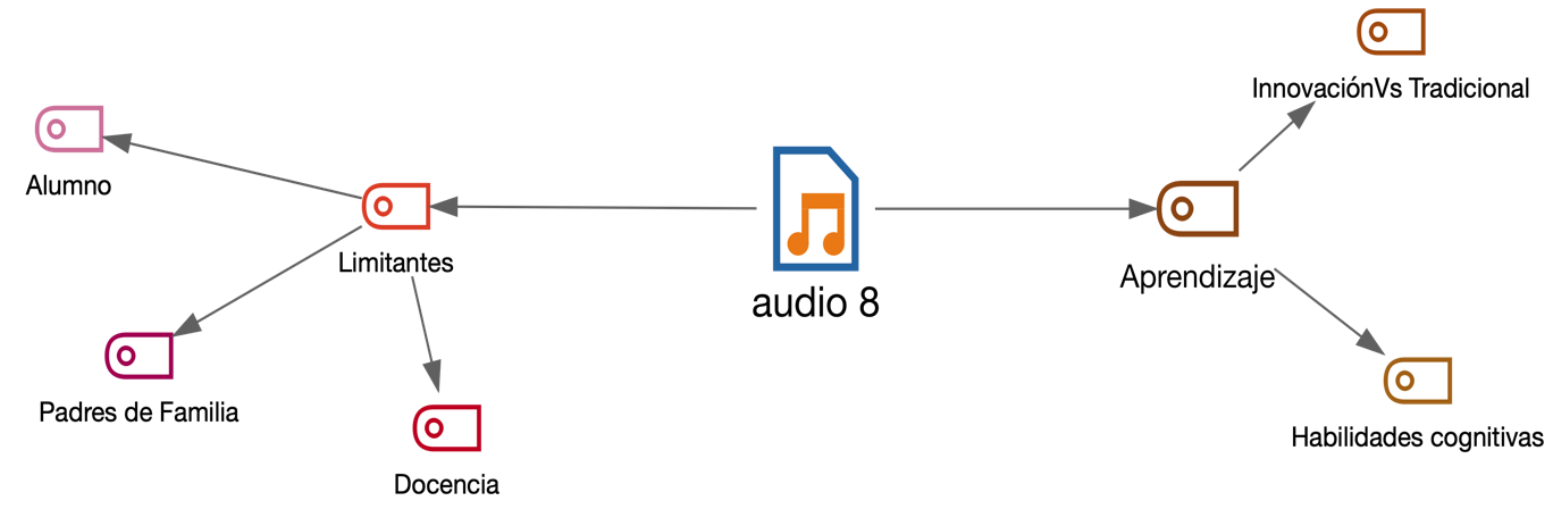

Fuente: Elaboración propia

En resumen, el directivo percibe el trabajo por proyectos como una estrategia innovadora, aunque con limitantes que impiden romper con esquemas educativos tradicionales implantados en docentes, estudiantes y padres de familia. Aun así, se reconoce que el implementar esta metodología de manera transversal en el programa de educación primaria ha propiciado el desarrollo de habilidades cognitivas en los estudiantes, por lo que progresivamente se va consolidando.

Por último, se realizó un comparativo entre las opiniones de los docentes y los estudiantes para determinar áreas de mejoría (p. ej., aprender a trabajar en equipo), así como sus bondades (p. ej., método motivador e inspirador que promueve el desarrollo de saberes), (figura 4). 


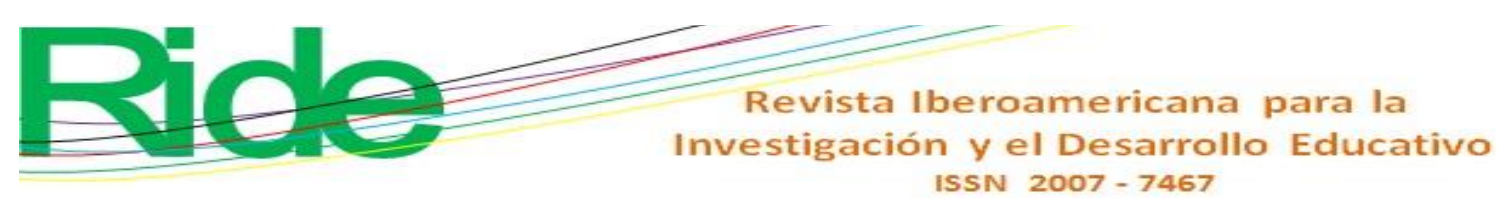

Figura 4. Modelo de contraste de casos entre entrevistas a docentes y estudiantes

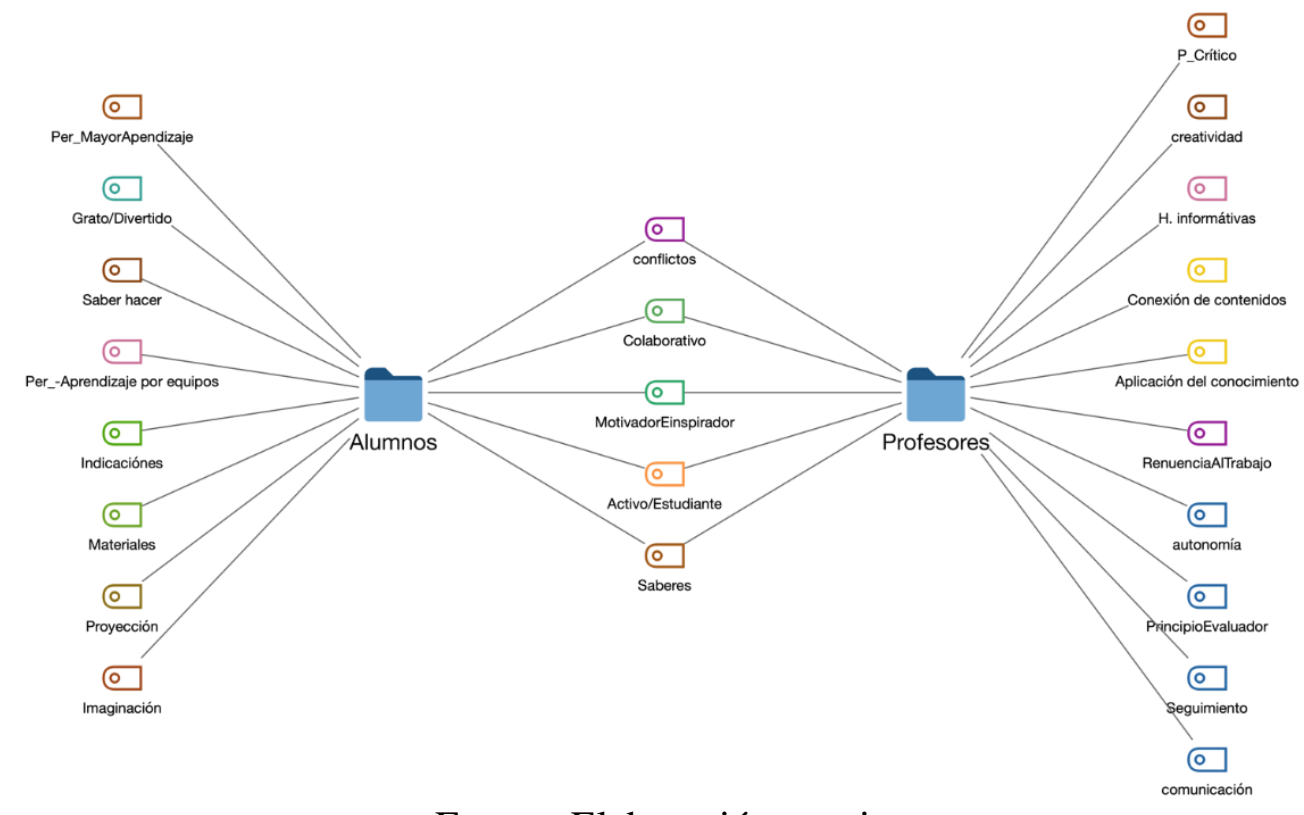

Fuente: Elaboración propia

Las coincidencias entre la percepción de los docentes y los alumnos se encuentran en el trabajo colaborativo favorable de la metodología, así como en su capacidad motivadora e inspiradora. Además, los participantes indican que la estrategia favorece el aprendizaje activo del estudiante y la construcción de sus saberes.

No obstante, también cabe señalar que los alumnos perciben que en ocasiones se requieren materiales para desarrollar las actividades, mientras que los docentes apuntan que la estrategia exige más compromiso y tiempo que el método tradicional, así como mejores principios de evaluación y seguimiento.

\section{Discusión}

Los resultados del estudio permiten caracterizar las bondades y los desafíos del aprendizaje basado en proyectos. En tal sentido, y como bondades, se reconoce que dicha metodología genera un ambiente de aprendizaje motivador, colaborativo, incluyente y lúdico. Mismos que concuerdan con Estos resultados concuerdan con Dvorak (2012) quien en su tesis demostró que el uso de la metodología por proyectos aumento la motivación autoinformada de los participantes principalmente en los constructos: autoeficacia, motivación intrínseca y trabajo en grupo como una experiencia positiva.

Otra ventaja tiene que ver con la manera en que la metodología fomenta la aplicación de los conocimientos y la generación de productos tangibles. De hecho, con esta estrategia se desarrollan la imaginación, la creatividad, la autonomía, el trabajo autónomo y el pensamiento crítico.

Estos resultados concuerdan con las afirmaciones de Muñoz y Díaz (2009), quienes mencionan que el trabajo por proyectos favorece la cooperación y ayuda a 


\section{Referencias}

Andréu, J. (2001). Las técnicas de análisis de contenido: una revisión actualizada. Recuperado de

http://mastor.cl/blog/wpcontent/uploads/2018/02/Andreu.-analisis-de-contenido.-34-pags-pdf.pdf

Aguerrondo, I. (1999). El nuevo paradigma de la educación para el siglo XXI. Organización de Estados Iberoamericanos para la Educación, la Ciencia y la Cultura. https://www.oei.es/historico/administracion/aguerrondo.htm

Araújo U. F. y Sastre, G. (2018). El aprendizaje basado en problemas. Barcelona, España: Editorial Gedisa.

Barba-Marín, R., Sonlleva Velasco, M. y García-Marín, N. (2018). Presencia, participación y progreso: el aprendizaje basado en proyectos en la trayectoria de una maestra en formación. Revista Electrónica Interuniversitaria de Formación del Profesorado, 21(2), 13-25. Doi: http://dx.doi.org/10.6018/reifop.21.2.323201

Bender, W. (2014). Penso, ed. Aprendizagem Baseada em Projetos: educação diferenciada para o século XXI. Porto Alegre, Brasil: Penso.

Cobo, G. y Valdivia, S. M. (2017). Aprendizaje basado en proyectos. Lima Perú: Pontificia Universidad Católica del Perú.

De la Torre Gamboa, M. (ed.) (2008). Concepciones y representaciones del cambio educativo. San Nicolás de los Garza: Facultad de Filosofía y Letras. Universidad Autónoma de Nuevo León.

Dewey, J. (1995). Democracia y educación. Una introducción a la filosofía de la educación. Madrid: Editorial Morata.

Díaz Barriga, F. (2006). Enseñanza situada. Vínculo entre la escuela y la vida. México D. F.: McGraw Hill.

Durán, M. M. (2012). El estudio de caso en la investigación cualitativa. Revista Nacional de Administración, 3(1), 121-134.

Dvorak, R. (2012). Motivation towards learning: A study of Alberni District Secondary School's Project Based Learning Grade 9 Program. Faculty of Education. Vancouver Island University. Retrieved from http://viuspace.viu.ca/bitstream/handle/10613/20 11/Dvorak.pdf?sequence=1

Fernández, M. C., Polo, Z., Fernández, C. J., Tallóns, S. R. y Hervás, M. T. (2018). "Voluntad de trabajo" dentro del aprendizaje basado en proyectos. Revista INFAD de Psicología. International Journal of Developmental and Educational Psychology, 2(1), 307-316. 
García Pérez, F. F. (2000). Los modelos didácticos como instrumento de análisis y de intervención en la realidad educativa. Revista Bibliográfica de Geografía y Ciencias Sociales, 207, 1-12.

García-Varcálcel, Muñoz-Repiso, A. y Gómez-Pablos, V. B. (2017). Aprendizaje basado en proyectos $(\mathrm{ABP})$ : evaluación desde la perspectiva de alumnos de Educación Primaria. Revista de Investigación Educativa, 35(1), 113-131. Doi: http://dx.doi.org/10.6018/rie.35.1.246811

Kilpatrick, W. (1918). The project method. The Teachers College Record, 19(4), 319335.

Larrañaga, A. (2012). El modelo educativo tradicional frente a las nuevas estrategias de aprendizaje (tesis de maestría). Bilbao, España: Universidad Internacional de La Rioja.

Martín, A. y Rodríguez, S. (2015). Motivación en alumnos de primaria en aulas con metodología basada en proyectos. Revista de Estudios e Investigación, 1, 1-5. Doi: 10.17979/reipe.2015.0.01.314

McAlpine, L. (2004): Designing learning as well as teaching. A research-based model for instruction that emphasizes learner practice. Active Learning in Higher Education, $5(2), 119-134$.

Molina, M. (2019). El aprendizaje basado en proyectos (ABP) en la formación metodológica del profesorado del grado de educación primaria. Enseñanza \& Teaching: Revista Interuniversitaria de Didáctica,37(1), 123-137. Doi: https://doi.org/10.14201/et2019371123137

Morales, P. B. y Landa, V. F. (2004). Aprendizaje basado en problemas. Theoría: Ciencia, Arte y Humanidades, 13, 145-15.

Muñiz, M. (2010). Estudios de caso en la investigación cualitativa. México: División de Estudios de Posgrado. Universidad Autónoma de Nuevo León. Facultad de Psicología. Recuperado de https://psico.edu.uy/sites/default/files/cursos/1_estudios-de-caso-en-lainvestigacion-cualitativa.pdf

Muñoz, A. M. y Díaz, M. P. (2009). Metodología por proyectos en el área de conocimiento del medio. Revista Docencia e Investigación, 9(1), 101-126.

Muñoz, J. y Maldonado, T. (2011). Aprendizaje con base en proyectos para desarrollar capacidades de problematización en educación superior. Actualidades Investigativas en Educación, 11(1), 1-19. 
Ortiz, A. O. (2011). Hacia una nueva clasificación de los modelos pedagógicos: el pensamiento configuracional como paradigma científico y educativo del siglo XXI. Praxis, 7(1), 121-137. Doi: https://doi.org/10.21676/23897856.18

Palomares, P. R. (2017). Desarrollo competencial en educación infantil a través del aprendizaje basado en proyectos en centros educativos de Jaén (tesis doctoral). Madrid: Universidad Camino José Cela.

Perrenoud, P. (1999). Apprendre à l'école à travers des projets : pourquoi ? comment ? Revista de Technología Educativa, 14(3), 311-321.

Piaget, J. (1977). The development of thought: Equilibrium of cognitive structures. New York: Viking Press.

Prieto, L. N. (2006). Aprendizaje activo en el aula universitaria: el caso del aprendizaje basado en problemas. Miscelánea Comillas. Revista de Ciencias Humanas y Sociales, 64(124), 173-196.

Quiroz Lima, M. E. (2002). La metodología por proyectos del PEP 92 y su influencia en la práctica docente. Recuperado de http://200.23.113.51/pdf/18400.pdf

Rekalde, I. R. y García, J. V. (2015). El aprendizaje basado en proyectos: un constante desafío. Innovación Educativa, (25), 219-234.

Rodríguez-Sandoval, E., Vargas-Solano, E. M. y Luna-Cortés, J. (2010). Evaluación de la estrategia "aprendizaje basado en proyectos". Educación y Educadores, 13-25.

Sanmartí, N. y Márquez, C. (2017). Aprendizaje de las ciencias basado en proyectos: del contexto a la acción. Ápice. Revista de Educación Científica, 1(1), 3-16. Doi: https://doi.org/10.17979/arec.2017.1.1.2020

Secretaría de Educación Pública [SEP] (1992). Programa de educación Preescolar 1992. México D. F.: SEP.

Secretaría de Educación Pública [SEP] (2011). Plan de estudios 2011. Educación básica. México D. F.: SEP.

Secretaría de Educación Pública [SEP] (2017). Modelo educativo para la educación obligatoria. México D. F.: SEP.

Silberman, M. (2006). Aprendizaje activo: 101 estrategias para enseñar. Argentina: Editorial Troquel.

Trujillo, F. (2015). Aprendizaje basado en proyectos. Infantil, primaria y secundaria. Madrid, España: Ministerio de Educación, Cultura y Deporte.

Vygotsky, L. S. (1962). Thought and language. Cambridge: MIT Press.

Wesley , H. y Richard P. (2004). Ideas prácticas para promover el aprendizaje activo y cooperativo: 27 maneras prácticas para mejorar la instrucción. Basado en los conceptos y principios del pensamiento crítico. Recuperado de 
http://www.launiversidadtecnologicadechile.cl/tportal/portales/tp4964b0e1bk10

2/uploadImg/File/TeoriaPractEns/PensamientoCritico/5_IdeasPracticasParaMejo rarInstruccion.pdf

\begin{tabular}{|l|l|}
\hline Rol de Contribución & Autor (es) \\
\hline Conceptualización & $\begin{array}{l}\text { María Obdulia González Fernández } \\
\text { Laura Becerra Vázquez } \\
\text { Grado de contribución: igual }\end{array}$ \\
\hline Metodología & María Obdulia González Fernández. \\
\hline Software & No aplica \\
\hline Validación & María Obdulia González Fernández \\
\hline Análisis Formal & María Obdulia González Fernández \\
\hline Investigación & Laura Becerra Vázquez \\
\hline Recursos & $\begin{array}{l}\text { Laura Becerra Vázquez (Apoyo) } \\
\text { María Obdulia González Fernández (Principal) }\end{array}$ \\
\hline Curación de datos & $\begin{array}{l}\text { Laura Becerra Vázquez } \\
\text { María Obdulia González Fernández } \\
\text { Grado de contribución: igual }\end{array}$ \\
\hline Escritura - Preparación del & $\begin{array}{l}\text { Laura Becerra Vázquez (Apoyo) } \\
\text { María Obdulia González Fernández (Principal) }\end{array}$ \\
\hline $\begin{array}{l}\text { Escritura - Revisión y } \\
\text { edición }\end{array}$ & $\begin{array}{l}\text { Laura Becerra Vázquez } \\
\text { María Obdulia González Fernández } \\
\text { Grado de contribución: igual }\end{array}$ \\
\hline Visualización & $\begin{array}{l}\text { María Obdulia González Fernández (Principal) } \\
\text { Laura Becerra Vázquez (Apoyo) }\end{array}$ \\
\hline Pdquisición de fondos & María Obdulia González Fernández \\
\hline Laura Becerra Vázquez \\
\hline María Obdulia González Fernández
\end{tabular}

